\title{
Performance Enhancement in P300 ERP Single Trial by Machine Learning Adaptive Denoising Mechanism
}

\author{
Syed Kamran Haider, Aimin Jiang, Muhammad Ali Jamshed, Haris Pervaiz and Shahid Mumtaz
}

\begin{abstract}
The P300-based lie detection scheme is yet another and advantageous tactic for unadventurous Polygraphy. In the proposed scheme, the raw electroencephalogram (EEG) signals are assimilated from 15 subjects during deception detection. After the assimilation, EEG signals are separated using an independent component analysis (ICA). The proposed adaptive denoising approach, extracts three kinds of features from denoised wave to reproduce $P 300$ waveform and identify the $P 300$ components at the $P_{z}$ electrode. Finally, in order to enhance the performance, four classifiers are used, i.e., support vector machine (SVM), linear discriminant analysis (LDA), k-nearest neighbor (KNN), and back propagation neural network (BPNN), achieving the accuracy of $74.5 \%, 79.4 \%, 97.9 \%$ and $89 \%$, respectively.
\end{abstract}

\section{INTRODUCTION}

D uring the past several decades, research into lie detection has gained much attention and gave directions to many moral, clinical, and legal fields. Lie detection based on P300, (an event-related potential (ERP) which is a component of electroencephalogram (EEG) waveform) can be further categorized into three groups, i.e., bootstrapped amplitude difference (BAD) method, bootstrapped correlation difference (BCD) method, and machine learning methods [1], [2]. In all the aforementioned methods, three stimuli, namely, Irrelevant (I) stimuli, Target $(\mathrm{T})$, and Probe $(\mathrm{P})$, have been displayed in front of the subjects [1].

However, the proper use of pattern recognition (PR) classifiers in lie detection scenario is not widely reported so far. In recent studies, functional MRI (fMRI) data support vector machine (SVM) dependent approach has been used to classify brain patterns for lie detection. Authors in [1] adopted the linear discriminant analysis (LDA) to examine the $P_{3}$ (the location of electrode placed on the parietal lobe of the head) behaviour and achieved the detection accuracy up to $86 \%$. The authors in [3] adopted SVM method for the case of deception detection in their research and attained the highest classification performance of $91.8 \%$.

To achieve the highest accuracy for the best lie detection method, it must contain a small number of stimuli [4]. Authors in [5] developed a strategy of using the SVM algorithm to identify lying and truth subjects. In addition to previous

S. K. Haider and A. Jiang are with College of Internet of Things Engineering, Hohai University Changzhou, China. M. A. Jamshed is with the Institute of Communication Systems (ICS), Home of 5G Innovation Centre (5GIC), University of Surrey, UK. H. Pervaiz is with the School of Computing and Communications, Lancaster University, UK. S. Mumtaz is with Campus Universitario de Santiago, Portugal. Email: kamranhaider85@yahoo.com, jiangam@hhuc.edu.cn,m.jamshed@surrey.ac.uk, h.b.pervaiz@lancaster.ac.uk and smumtaz@av.it.pt. studies, a few reports show the potential of machine learning methods for single trial-based lie detection [1], [2]. In these models, the single trial optimal features are extracted in order to train the classifiers and find the different brain behaviors. However, the superimposed noise on the signals in the single trial method degrades the lie detection performance.

The noise component is superimposed with single trials in the case of P300 feature extraction. In this scenario EEG waveform recorded by each sensor channel is divided into two parts. The first is extra-skull noise and the second one is intraskull noise generated at different brain parts, having effects on ERP and EEG signal recording. The ERP element cannot be denoted from sensors directly. Aforementioned approaches are not able to remove noise factor and irregular EEG components from P300 waveform because of their scalp projections and time occurrence overlapped with each other [6].

In this study, we present a novel adaptive signal denoising (ASD) method to overcome the shortcomings of the method outlined in [4], which is inefficient to boost the signal-to-noise ratio (SNR) of the waveform and selecting the least features. In our proposed study, after applying an independent component analysis (ICA), the ASD method can be used to improve the system efficiency and select appropriate features, which leads to improvement at the classification stage. In the ASD methodology, we consider more affecting areas which have strong P300 ERP waveform effects on $P_{3}, P_{4}, P_{z}, C_{z}$ and $O_{z}$ positions [7]. Likewise, the proposed ASD has the ability to choose P300 independent sources for the reconstruction of $P_{z}$ wave. Hence, the ASD is more reasonable and reliable than the aforementioned study presented in [4]. The objective of our research presented in this paper is to reduce the threshold issue and use fewer stimuli for fast processing.

\section{Machine Learning Based Methodology}

To overcome the noise effect and achieve the reconstructed waveform with better SNR for better classification of P300 components, the proposed ASD method is developed in this section. By adopting this training method we can determine the optimal criterion values in addition to the values of the ASD method, in explicit classifiers. Algorithm 1 gives a logical view of the proposed method. Using EEG signals of 14 channels as input at pre-processing and reconstruction leads to feature extraction stage. After extraction of features, the optimal features are selected and fed into four classifiers for their performance comparison. 


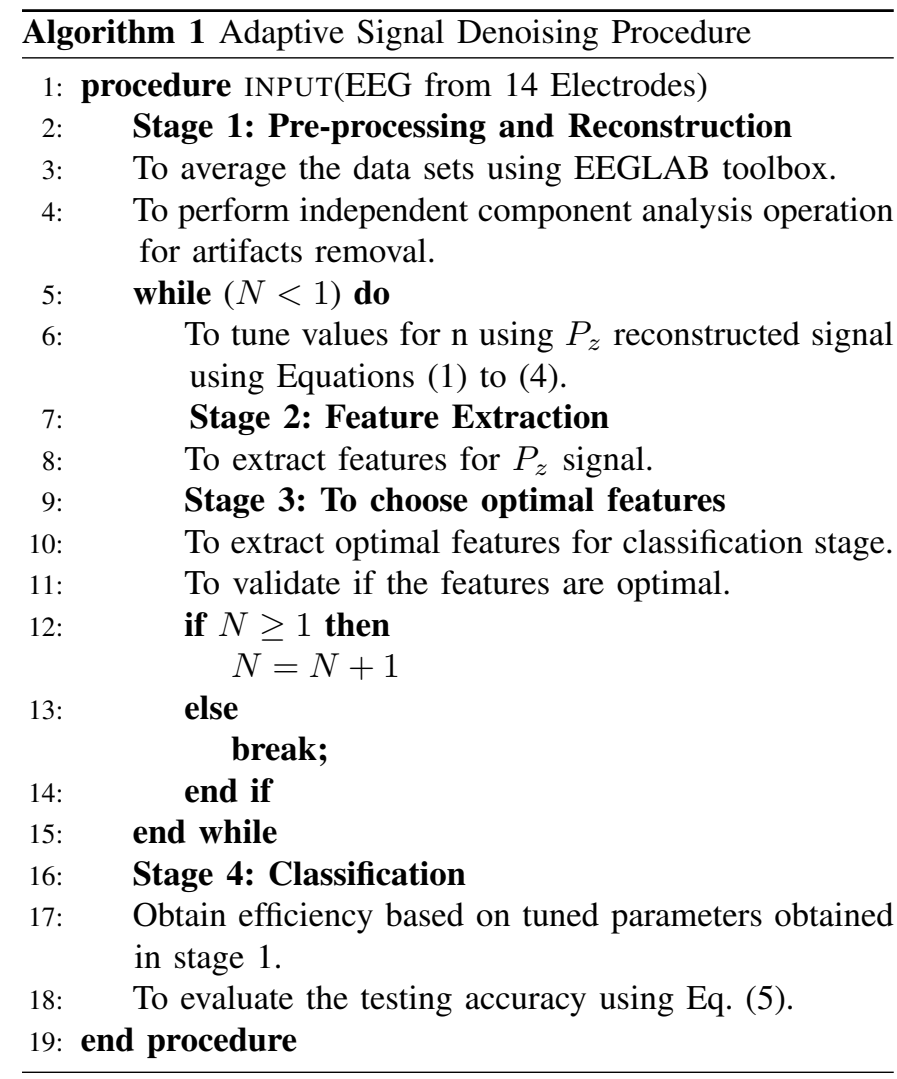

\section{A. EEG Data Acquisition}

The standard of 10-20 electrode placement is used in our study, which has twelve electrodes $\left(F_{P_{1}}, F_{P_{2}}, F_{3}, F_{z}, F_{4}\right.$, $\left.C_{3}, C_{z}, C_{4}, P_{3}, P_{z}, P_{4}, O_{z}\right)$. Vertical Electrooculography (VEOG) and horizontal Electrooculography (HEOG) signals are recorded from the right eye and outer canthus respectively. In order to filter the online EEG and EOG recorded signal, a bandpass filter with passband $0.1-30 \mathrm{~Hz}$ is utilized with the sampling frequency $500 \mathrm{~Hz}$. EEG signals recorded by all the sensors are grounded to right side of earlobe. The sensor impedance can't exceed $2 \mathrm{k} \Omega$.

\section{B. Pre-Processing}

To remove the ocular artifacts, EEG signals are segmented into epoched datasets using EEGLAB toolbox, each having a duration from $0.10 \mathrm{~s}$ to $1.20 \mathrm{~s}$ after the stimulus onset. Then SCAN software of Neuroscan is used to remove the ocular artifacts [8] of each set, i.e., those datasets having voltage more than $+75 \mathrm{mV}$ are discarded from the datasets that contained single trail. For each subject group, datasets referred to probe response and were selected to take average of each 5 datasets and taken into account as one single average set.

\section{Adaptive Signal De-Noising for P300 Improvement}

Each dataset is first decomposed by ICA, resulting in decomposed independent components (ICs) and a matrix $P^{-1}$. Here the ICA uses extended INFOMAX algorithm, also known as extended independent component analysis (EICA), to allow sub-Gaussian distributions [9], [10]. A precise decomposition of multi-channeled EEG signals can be obtained by EICA respectively. It has been found that P300 has the smallest values at $F_{z}$, intermediate at $C_{z}$, and the largest at $P_{z}$ [11]. The ASD algorithm is divided into 4 steps. Firstly, the normalization of matrix $P^{-1}$ is performed using the methodology defined in [4] as follow:

$$
V_{l j}=\frac{\left|P_{l j}^{-1}\right|}{\max \left(\left|P_{. j}^{-1}\right|\right)},
$$

where $l=1,2, \ldots .14, j=1,2, \ldots .14$ and notation $\|$ represents an absolute computation. New EEG, can be denoted by $X^{\prime}(t)$ and given as follows:

$$
X^{\prime}(t)=V D(t)=\left[\begin{array}{ccccc}
v_{11} & \ldots & v_{1 j} & \ldots & v_{1 n} \\
\vdots & \ldots & \vdots & \ldots & \vdots \\
v_{l 1} & \ldots & v_{l j} & \ldots & v_{l n} \\
\vdots & \ldots & \vdots & \ldots & \vdots \\
v_{n 1} & \ldots & \vdots & \ldots & v_{n n}
\end{array}\right]\left[\begin{array}{c}
d_{1 t} \\
\vdots \\
d_{j t} \\
\vdots \\
d_{n t}
\end{array}\right]
$$

Secondly, the presumed sequence number of electrodes i.e., $P_{z}, P_{3}, P_{4}, C_{z}$ and $O_{z} . G_{j}$ are calculated as:

$\mathbf{v}_{P_{z}}(t)=\mathbf{v}_{P_{z} j}+\omega_{1} \times\left(\mathbf{v}_{P_{3} j}+\mathbf{v}_{P_{4} j}\right)+\omega_{2} \times \mathbf{v}_{C_{z} j}+\omega_{3} \times \mathbf{v}_{O_{z} j}$,

where $\omega_{1}, \omega_{2}$ and $\omega_{3}$ are the parameters on different weighted elements $\mathbf{v}_{l j}$. In the above equation, $G_{j}$ represents the integrated scattered energy on different responsive positions of brain from $j^{\text {th }}$ independent component (IC). The maximum $G_{j}$ is the highest liability in the $j^{\text {th }}$ IC of the P300 ICs.

Thirdly, for the sorting of index vector $F$ and the vector $E$, the 14 values in $G=\left\{G_{1}, G_{2}, G_{3}, \ldots . . G_{14}\right\}$ are arranged systematically in a descending pattern. The key index vector $F$ gives $F_{j}$ an element position in vector $G$. Fourthly, the back projection is estimated using the following formula:

$$
Y_{P_{z}}(t)=\sum_{j=1}^{n} P_{P_{z} F_{j}}^{-1} \times D_{F_{j}}(t),
$$

where $n=$ number of P300 ICs, $Y_{P_{z}}(t)=$ reconstructed wave.

\section{Features Selection and Extraction}

After the reconstruction of the P300 signal with the highest SNR by using the ASD model, the time-domain and the frequency-domain were analyzed and three groups of features were extracted from $P$ response (response signal from $P$ stimuli). Many researches have still on the way related to this methodology, such as [1], [12]. By the feature extraction stage, two optimal feature sample sets, were obtained with the class label being -1 and 1 from innocent and guilty, respectively. Each featured sample contains the morphological, frequency and wavelet frequency based values respectively [1]. After that each feature value was normalized to $[-1,1]$ before classification.

\section{E. Classification Methods}

To obtain the best classification results is by selecting the optimal features from the hybrid model and integrating it with the features extraction stage of the proposed method. In addition, four independent classification methods such as LDA, back propagation neural network (BPNN), SVM and k-nearest neighbor $(\mathrm{KNN})$ are also investigated. The optimum recorded features of sample datasets was extracted from two classes in 
TABLE I: Performance of each classifiers based on F-score method [4]

Sensitivity/Specificity [\%]

\begin{tabular}{c|c|c}
\hline Classifier Models & Training & Testing \\
\hline SVM & $91.00+1.80 / 90.98+1.85$ & SVM \\
\hline BPNN & $79.27+1.66 / 78.78+1.72$ & BPNN \\
\hline FDA & $68.38+2.13 / 67.22+1.94$ & FDA \\
\hline
\end{tabular}

[13] to perform subject-wise cross-validation (SWCV). For every individual datastes, samples extracted from all the subjects were categorized into training and testing sets. In the proposed method of lie detection technique, optimal parameters must be set for two groups which is described below:

- ASD parameters: $\omega_{1}, \omega_{2}, \omega_{3}, n$.

- The recommended hyper parameters for respective classifier.

The parameters in ASD bring change in the optimal values of the hyper parameters. By using multi-dimension grid searching approach, a simultaneous tuning of the two parameter groups is performed. Recommended values by the EEG expert consider to tune the parameters as: the variation of $\omega_{1}, \omega_{2}$ and $\omega_{3}$ with the range of 0.2 to 1 and a step size of 0.15 is being done, the $n$ values varies from 1 to 14 . For the parameter adjustment in BPNN case, the learning rate $\boldsymbol{g}$ and sigmoid hidden nodes $\boldsymbol{a}$ is tuned (0.002 value set as control precision) [4].

\section{Simulation Setup}

The goal of this study is to propose an algorithm which can easily distinguish innocent and guilty subjects using a minimum number of probes. For all simulation related scenario and parameters online public repository DRYAD are available without any restrictions and limitations [14]. The authors in [4] have used spatial denosing algorithm in preprocessing, perform feature extraction and then use F-score method for selection of optimum features. Table I describes the performance of the three classifiers, i.e., fisher discrimination analysis (FDA), SVM, and BPNN respectively of the proposed prior method mentioned in [4]. The aim of this proposed method is to improve the system performance in contrast to the method proposed in [4]. In order to evaluate the performance of our proposed machine learning based ASD procedure as mentioned in algorithm 1, the simulation results are evaluated in comparison to the benchmark performance outlined in [4]. The mathematical expression in Eq. (5) is used to calculate the results in Table I and Table II respectively. From Table I, we can observe that FDA is obviously lower than that of SVM and BPNN. This result demonstrates that the data attained from two subjects while doing lie detection cannot be linearly separated whereas SVM is extensively greater than BPNN.

$$
\text { Accuracy }[\%]=\frac{M_{\text {sen }} \pm S D_{\text {sen }}}{M_{\text {spe }} \pm S D_{\text {spe }}} \text {. }
$$

In our proposed technique, we find all possible optimum features. Before feature extraction we develop a new denoising technique called ASD, which shows significant improvement in results. Especially the LDA results are much improved by the proposed denoising algorithm. In the following section we will discuss about the guilty/innocent subjects, before and after ICA and the reconstruction of $P_{z}$ wave for guilty and innocent
TABLE II: Performance enhancement of proposed machine learning based adaptive denoising procedure

\begin{tabular}{c|c|c}
\multicolumn{3}{c}{ Sensitivity/Specificity [\%] } \\
\hline Classifier Models & Training & Testing \\
\hline SVM & $97.90+1.61 / 97.40+1.34$ & SVM \\
\hline BPNN & $89+2.1 / 88.2+2.06$ & BPNN \\
\hline LDA & $74.5+2.04 / 73.6+1.94$ & FDA \\
\hline KNN & $79.4+2.17 / 78.6+1.99$ & KNN \\
\hline
\end{tabular}

subject from ASD. We are using 14 electrodes data, electrodes names are $F_{P_{1}}, F_{P_{2}}, F_{3}, F_{z}, F_{4}, C_{3}, C_{z}, C_{4}, P_{3}, P_{z}, P_{4}, O_{z}$, VEOG, HEOG names are in sequence. After ICA we apply ASD, a proposed denoising technique for better improvement of results. In ASD we reconstruct the average $P_{z}$ wave for both classes, innocent, and guilty.

\section{Performance Evaluation}

The reconstruction of $P_{z}$ is depicted in Fig. 1. The single trial (blue) and respective averaged waveform (red) for innocent subject before applying ASD are shown in Fig. 1(a). Similarly Fig. 1(b) shows single trial (blue) and respective averaged waveform (red) for guilty subject before ASD and Fig. 1(c) shows the reconstructed waveform for innocent (blue) and guilty (red) subject after applying ASD. After ASD algorithm we perform wavelet decomposition [1] on selected $P_{z}$ wave, we use wavelet coefficients of lowpass and highpass filter, as a feature.

The performance of our proposed mechanism is evaluated in terms of four classifiers resulting in significantly improved

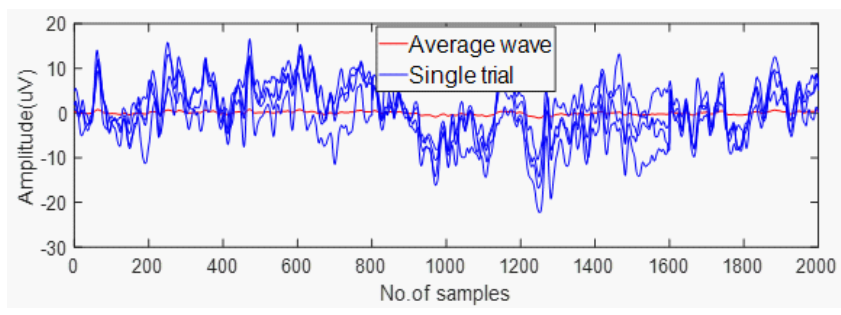

(a)

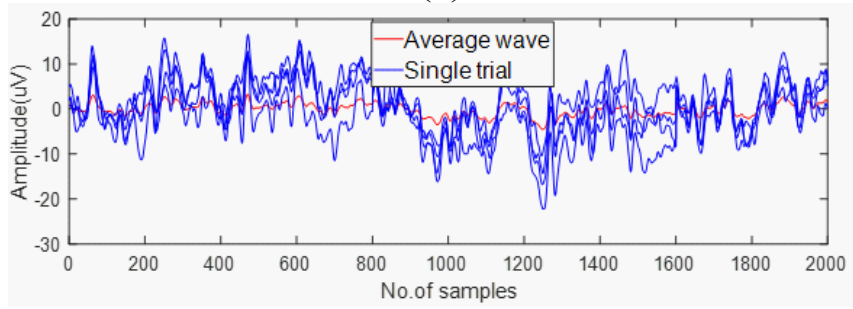

(b)

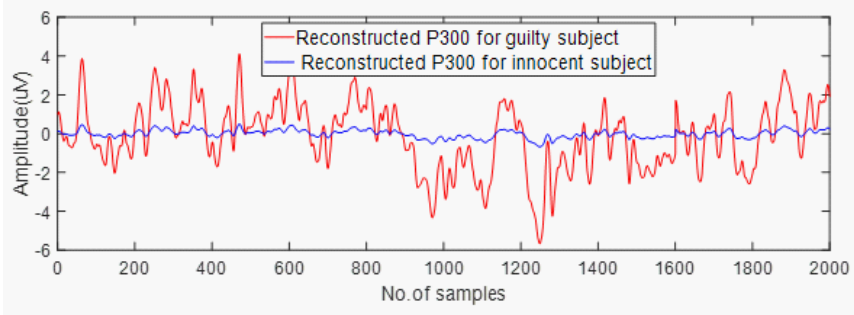

(c)

Fig. 1: Reconstructed waveform after applying ASD on guilty and innocent subjects. 
results in comparison to the traditional method using $\mathrm{F}$ score technique in Table I [4]. In comparison to Table I, the Table II illustrates the significant improvement in performance achieved by the denoising algorithm. We can clearly observe that how our system improves the result of classifiers with previous implemented methods. Fig. 2 and Fig. 3 shows the graphs of sensitivity and specificity of each subject after selection of suitable denoising algorithm. In this proposed implemented method, the noise factor in single trial P300 ERP wave can be further sub-categorized as follow:

- Ill-assorted feedback to specific kind of stimulus, which can effect in proper detection of cognitive state [3].

- Other one is usual noise occurs during EOG artifacts. Therefore, prior to the implementation of proposed machine learning based ASD model, each 5 raw EEG epoched datasets are averaged to reduce the effect of noise components on the SNR of P300 ERP wave. This can help us to increase the efficiency and reliability of the whole lie detection system. This intended approach at pre-processing level in the case of lie detection is not limited as it has been done by the authors in [3].

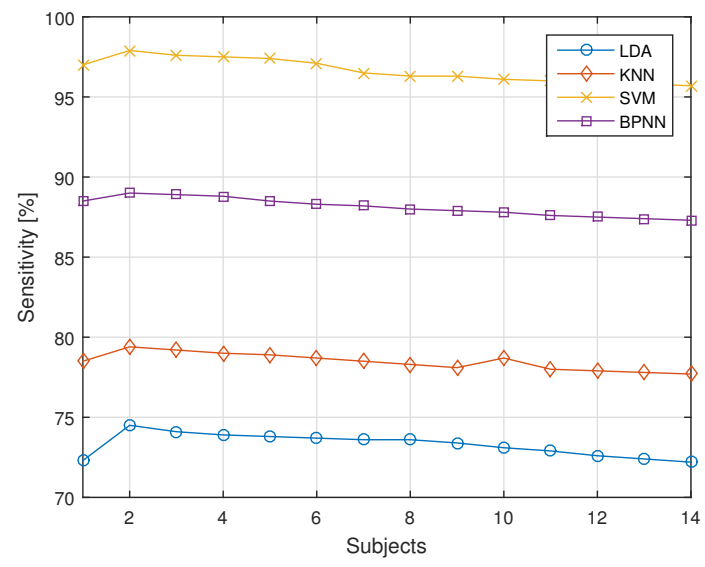

Fig. 2: Sensitivity of P300 ERP component for the each subject after selection of ASD algorithm.

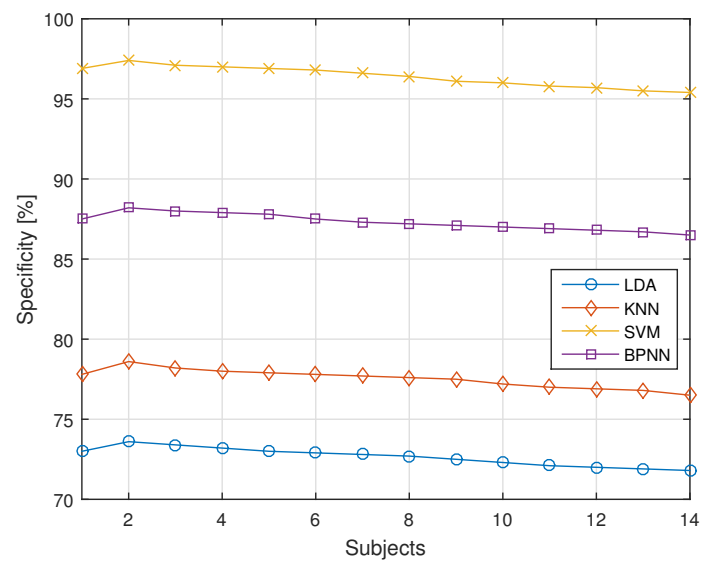

Fig. 3: Specificity of non-P300 ERP component for the each subject after selection of ASD algorithm.

\section{CONCLUSION}

To make the lie detector time effective, few stimuli is preferred and is important for many practical applications. In this paper, a novel ICA based on ASD is proposed to enhance the SNR. Furthermore, to distinguish the guilty subjects from those of innocent, the machine learning techniques i.e. LDA, SVM, KNN, BPNN are employed. To reduce the noise effect on the performance of lie detector, the ASD algorithm is proposed, that separates the P300 components from noise signals. A new $P_{z}$ wave was constructed, having more obvious features of P300. It is acclaimed that meanwhile extracting the features of $P_{z}$ wave 14 channel electrodes were practiced to apply ICA for the effectiveness of ICA and ASD algorithms. While in pre-processing phase ICA can also help in removing the ocular objects, which serves as it's advantage. Moreover, our results show a satisfying improvement in the performance enhancement comparison to the previous methods shown in Table II.

\section{REFERENCES}

[1] V. Abootalebi, M. H. Moradi, and M. A. Khalilzadeh, "A new approach for EEG feature extraction in P300-based lie detection," Computer methods and programs in biomedicine, vol. 94, no. 1, pp. 48-57, 2009.

[2] V. Abootalebi, M. H. Moradi, and M. A. Khalilzadeh, "A comparison of methods for erp assessment in a P300-based GKT,' Int J Psychophysi, vol. 62, no. 2, pp. 309-320, 2006

[3] J. Gao, X. Yan, J. Sun, and C. Zheng, "Denoised P300 and machine learning-based concealed information test method," Computer Methods and Programs in Biomedicine, vol. 104, no. 3, pp. 410-417, 2011.

[4] J. Gao, H. Tian, Y. Yang, X. Yu, C. Li, and N. Rao, "A novel algorithm to enhance P300 in single trials: Application to lie detection using F-Score and SVM," PLOS one, vol. 9, no. 11, pp. 1-15, 2014.

[5] A. I. Simbolon, A. Turnip, J. Hutahaean, Y. Siagian, and N. Irawati, "An experiment of lie detection based EEG-P300 classified by SVM algorithm.," in IEEE International Conference on Automation, Cognitive Science, Optics, Micro Electro-Mechanical System, and Information Technology (ICACOMIT), pp. 68-71, 2015.

[6] T.-P. Jung, S. Makeig, M. Westerfield, J. Townsend, E. Courchesne, and T. J.Sejnowski, "Removing of eye activity artifacts from visual eventrelated potentials in normal and clinical subjects," Clinical Neurophysiology, vol. 111, no. 10, pp. 1745-1758, 2000.

[7] W. Dhanapala, A. Bakmeedeniya, S. Amarakeerthi, P. Jayaweera, and S. Sumathipala, "A brain signal-based credibility assessment approach," in Fuzzy Systems Association and 9th International Conference on Soft Computing and Intelligent Systems (IFSA-SCIS), 2017 Joint 17th World Congress of International, pp. 1-6, IEEE, 2017.

[8] J. F. Gao, Y. Yang, P. Lin, P. Wang, and C. X. Zheng, "Automatic removal of eye-movement and blink artifacts from EEG signals," Brain topography, vol. 23, no. 1, pp. 105-114, 2010.

[9] T.-P. Jung, C. Humphries, T.-W. Lee, S. Makeig, M. J. McKeown, V. Iragui, and T. J. Sejnowski, "Extended ICA removes artifacts from electroencephalographic recordings," in Advances in neural information processing systems, pp. 894-900, 1998.

[10] T.-W. Lee, M. Girolami, and T. J. Sejnowski, "Independent component analysis using an extended infomax algorithm for mixed subgaussian and supergaussian sources," Neural computation, vol. 11, no. 2, pp. 417-441, 1999.

[11] J. Rosenfeld, J. W.Ellwanger, K. Nolan, S. Wu, R. G.Bermann, and J. Sweet, "P300 scalp amplitude distribution as an index of deception in a simulated cognitive deficit model," Int J Psychophysi, vol. 33, no. 1, pp. 3-19, 1999.

[12] W.-Y. Hsu, C.-C. Lin, M.-S. Ju, and Y.-N. Sun, "Wavelet-based fractal features with active segment selection: Application to single-trial EEG data," Journal of neuroscience methods, vol. 163, no. 1, pp. 145-160, 2007.

[13] S.-Y. Shao, K.-Q. Shen, C. J. Ong, E. P. V. Wilder-Smith, and X.-P. Li, "Automatic EEG artifact removal: A weighted support vector machine approach with error correction," IEEE Trans on Biomed Eng, vol. 56, no. 2, pp. 336-344, 2009.

[14] J. Gao, H. Tian, Y. Yang, X. Yu, C. Li, and N. Rao, "Data from: A novel algorithm to enhance P300 in single trials: application to lie detection using F-score and SVM," 2014. 